\title{
PET imaging in patients with brain metastasis-report of the RANO/PET group
}

\author{
Norbert Galldiks, Karl-Josef Langen, Nathalie L. Albert, Marc Chamberlain, Riccardo Soffietti, \\ Michelle M. Kim, lan Law, Emilie Le Rhun, Susan Chang, Julian Schwarting, Stephanie E. Combs, \\ Matthias Preusser, Peter Forsyth, Whitney Pope, Michael Weller, and Jörg C. Tonn
}

\begin{abstract}
Department of Neurology, University Hospital Cologne, Cologne, Germany (N.G.); Institute of Neuroscience and Medicine 3, 4, Research Center Juelich, Juelich, Germany (N.G., K-J.L.); Center of Integrated Oncology, Universities of Cologne and Bonn, Cologne, Germany (N.G.); Department of Nuclear Medicine, University Hospital Aachen, Aachen, Germany (K-J.L.); Department of Nuclear Medicine, Ludwig Maximilians-University of Munich, Munich, Germany (N.L.A.); Departments of Neurology and Neurological Surgery, Fred Hutchinson Cancer Research Center, University of Washington, Seattle, Washington, USA (M.C.); Department of Neuro-Oncology, University and City of Health and Science Hospital, Turin, Italy (R.S.); Department of Radiation Oncology, University of Michigan, Ann Arbor, Michigan, USA (M.M.K.); Department of Clinical Physiology, Nuclear Medicine and PET, Rigshospitalet, University of Copenhagen, Denmark (I.L.); Department of Neurosurgery, University Hospital Lille, Lille, France (E.L.R.); Department of Neurosurgery, University of California, San Francisco, California, USA (S.C.); Department of Neurosurgery, Ludwig Maximilians-University of Munich, Munich, Germany (J.S., J.C.T.); German Cancer Consortium, Partner Site Munich, Germany (J.S., J.C.T.); Department of Radiation Oncology, Technical University Munich, Munich, Germany (S.E.C.); Department of Medicine I and Comprehensive Cancer Centre CNS Tumours Unit, Medical University of Vienna, Vienna, Austria (M.P.); Moffitt Cancer Center, University of South Florida, Tampa, Florida, USA (P.F.); Department of Radiological Sciences, David Geffen School of Medicine, University of California, Los Angeles, California , USA (W.P.); Department of Neurology, University Hospital and University of Zurich, Zurich, Switzerland (M.W.)
\end{abstract}

Corresponding Author: Norbert Galldiks, M.D., Institute of Neuroscience and Medicine (INM-3), Research Center Juelich, Leo-Brandt-St. 5, 52425 Juelich, Germany (n.galldiks@ fz-juelich.de) and Dept. of Neurology, University Hospital Cologne, Kerpener St. 62, 50937 Cologne, Germany (norbert.galldiks@uk-koeln.de).

\begin{abstract}
Brain metastases (BM) from extracranial cancer are associated with significant morbidity and mortality. Effective local treatment options are stereotactic radiotherapy, including radiosurgery or fractionated external beam radiotherapy, and surgical resection. The use of systemic treatment for intracranial disease control also is improving. BM diagnosis, treatment planning, and follow-up is most often based on contrast-enhanced magnetic resonance imaging (MRI). However, anatomic imaging modalities including standard MRI have limitations in accurately characterizing posttherapeutic reactive changes and treatment response. Molecular imaging techniques such as positron emission tomography (PET) characterize specific metabolic and cellular features of metastases, potentially providing clinically relevant information supplementing anatomic MRI. Here, the Response Assessment in NeuroOncology working group provides recommendations for the use of PET imaging in the clinical management of patients with BM based on evidence from studies validated by histology and/or clinical outcome.
\end{abstract}

\section{Keywords}

amino acid PET | brain metastases | FDG PET | FET | pseudoprogression

Brain metastases (BM) often occur in advanced malignancies but may also be an initial disease manifestation in, for example, CUP (cancer of unknown primary). BM derive most frequently from lung cancer (40-50\% of all BM), breast cancer (15-20\%), melanoma (5-20\%), renal cancer $(5-10 \%)$, and cancers of the gastrointestinal tract (5\%). ${ }^{1}$ The prognosis of patients with $\mathrm{BM}$ is usually poor, with a general median survival of several weeks in untreated patients and up to several months following 
oncological treatment. Some molecularly defined patient subsets, such as those positive for human epidermal growth factor receptor 2 breast cancer or anaplastic lymphoma kinase non-small cell lung cancer, may demonstrate significantly longer survival. Outcomes continue to improve with advances in systemic and regional therapy.

Regional treatment options for BM are neurosurgical resection, radiotherapy (eg, radiosurgery, fractionated external beam radiotherapy), and combinations thereof. ${ }^{2}$ Systemic treatment options such as targeted therapy and immunotherapy that can control both intracranial and extracranial disease are improving. ${ }^{3}$ Depending on the number of $\mathrm{BM}$ and the performance status of the patient, radiotherapy is an effective treatment of $\mathrm{BM}$, either as whole-brain radiation therapy (WBRT) or, increasingly, as stereotactic radiosurgery (SRS). ${ }^{4}$ Furthermore, surgery is frequently combined with postoperative radiotherapy, particularly in patients with single BM or oligometastatic brain disease. ${ }^{5}$ Despite various treatment options, BM recurrence is common.

Contrast-enhanced magnetic resonance imaging (MRI) is the cornerstone of metastatic brain tumor evaluation. This technique has widespread availability and excellent spatial resolution, but can exhibit low specificity, resulting in substantial diagnostic challenges. ${ }^{6-8}$ These challenges include discriminating BM from potential mimics that also demonstrate nodular or ring enhancement. Furthermore, MRI signal abnormalities-including T2 or fluid attenuated inversion recovery (FLAIR) hyperintensity, newly diagnosed contrast-enhancing lesions, or an increase in the extent of contrast enhancement-are nonspecific findings that can be caused by a variety of entities, including infection, inflammation, ischemia, demyelination, and treatment-related effects. In particular, reactive changes after surgery, radiotherapy, and systemic drug treatment can be difficult to distinguish from true disease relapse. This phenomenon of worsening treatment-related changes mimicking progression, termed pseudoprogression, is of clinical importance as potentially effective treatment might be erroneously terminated prematurely. 9,10 Pseudoprogression is a concern not only following radiation-based therapies, but also following immunotherapy, where a not well-characterized subset of patients manifests delayed response to therapy or therapy-induced inflammation that can simulate progressive disease. ${ }^{11,12}$

Successful management of patients with BM relies on accurate and early assessment of treatment response. The ability to predict or quickly detect lack of response to treatment may enable the early discontinuance of a particular therapy, thereby preventing additional toxicity and allowing for the earlier initiation of alternative therapy. Despite promising efforts in defining response criteria for $\mathrm{BM},{ }^{10,13}$ limitations remain, particularly with newer systemic treatment options such as targeted therapy and immunotherapy. Modalities which provide additional information on tumor physiology, including metabolism and proliferation, are increasingly applied problem-solving tools in patients with BM.

One promising method to investigate tumor physiology is positron emission tomography (PET). PET uses a variety of radioactive tracers that target various metabolic and molecular processes. PET imaging can provide relevant additional information that enables improved disease assessment, especially in clinically equivocal situations. Notwithstanding other PET tracers, the use of PET with radiolabeled amino acids, in particular, has been validated as an important diagnostic tool in brain cancer. ${ }^{6,14-16}$ The overexpression of L-type amino acid transporters (LATs) in BM makes intracranial metastases a compelling target for amino acid PET imaging. ${ }^{17}$

In this review, the Response Assessment in NeuroOncology (RANO) working group provides evidence-based recommendations for the use of PET imaging using tracers of amino acid transport and other targets, including glucose metabolism, in the management of patients with BM.

\section{Search Strategy, Selection Criteria, and Levels of Validation}

A PubMed search of the published literature was performed with the combination of the search terms "brain metastasis/metastases," "PET," "positron," “FDG," "amino acid," “methionine," “FET," “FDOPA," “FLT," “TSPO," “PSMA," "radiotherapy," "radiation-induced changes/radiation injury," "radionecrosis," "pseudoprogression," "treatment monitoring," "assessment of treatment response," and "immunotherapy" prior to and inclusive of August 2018. Additionally, articles identified through searches of the authors' own files were included in the search. Results of the search were evaluated by the working group with respect to the level of evidence and the grade of validation of the PET studies examined. As described previously, ${ }^{14,15}$ any study that correlated PET findings with histopathology was considered to represent the highest degree of validation. Next, correlation with $\mathrm{MRI}$ and with the patient's clinical course was considered the second level of validation. Only papers constituting levels 1-3 evidence according to the Oxford Centre for Evidence-based Medicine (the Oxford 2011 Levels of Evidence) were included. In brief, a randomized controlled trial fulfills the criteria for Oxford level 1, a prospective cohort study corresponds to Oxford level 2, and a retrospective study is consistent with Oxford level 3.

\section{PET Tracers for Imaging of Patients with Brain Metastasis}

In the following paragraphs, available PET tracers which address various pathophysiological pathways or molecular entities in BM are summarized.

\section{Glucose PET}

${ }^{18} \mathrm{~F}$-2-fluoro-2-deoxy-D-glucose (FDG) represents the most widely used tracer in oncologic PET imaging and has evolved over the last several decades into the paramount clinical PET modality for cancer detection. ${ }^{18}$ Due to the long half-life of the fluorine-18 isotope (110 minutes), inhouse production of this tracer is not necessary, overcoming logistic problems that occur with isotopes of shorter half-life. Thus, FDG can be transported to all PET centers, alleviating the need for on-site cyclotron-based manufacturing. Increased FDG uptake is commonly seen in highly proliferating cancer cells because of increased expression of glucose transporters and hexokinase, the enzyme that converts glucose (and FDG) to a phosphorylated 
product. Related to increased glycolysis, the uptake of FDG in neoplastic tissue is generally higher than in nonneoplastic tissue. However, the high and regionally variable FDG uptake in normal brain parenchyma often makes the delineation of tumors in the brain difficult ${ }^{14}$ (Fig. 1). Furthermore, inflammatory tissue can exhibit high FDG tracer uptake, also diminishing diagnostic specificity. ${ }^{6}$

\section{Amino Acid PET Tracers}

For decades, radiolabeled amino acids have been used in neuro-oncological practice. ${ }^{19}{ }^{11} \mathrm{C}$-methyl-L-methionine (MET), an essential amino acid labeled with the isotope carbon-11, has been the most commonly employed. ${ }^{18,20}$ The relatively short half-life of carbon-11 (20 minutes) limits its use to PET facilities with an on-site cyclotron. Consequently, other amino acids labeled with the positron-emitting isotope ${ }^{18} \mathrm{~F}$, which has a half-life of 110 minutes, have been developed, resulting in improved distribution, efficiency, and cost-effectiveness. ${ }^{21}$ For example, $O-\left(2-\left[{ }^{18} \mathrm{~F}\right]\right.$ fluoroethyl)L-tyrosine (FET) was developed in the late 1990s, and its use has grown rapidly, particularly in western Europe, in recent years. ${ }^{22-24}$ Clinical results in glioma patients with PET using FET appear to be comparable to $\mathrm{MET}^{25-27}$ In 2014, FET was approved as a medical drug in Europe (Switzerland). ${ }^{28}$

Another ${ }^{18} \mathrm{~F}$-labeled amino acid analogue is 3,4-dihydroxy$6-\left[{ }^{18} \mathrm{~F}\right]$-fluoro-L-phenylalanine (FDOPA), which was initially developed to evaluate dopamine synthesis in the basal

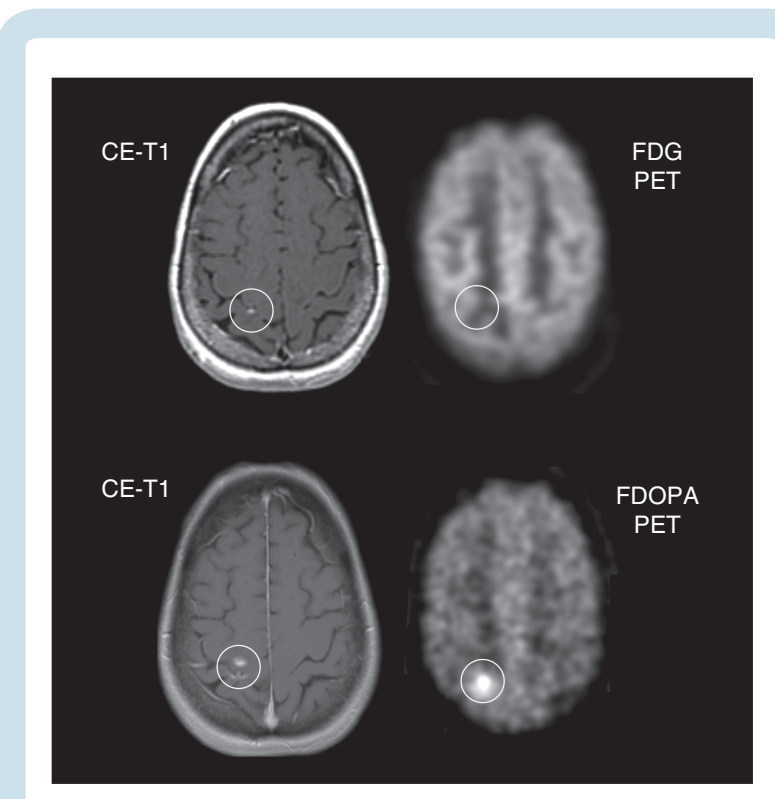

Fig. 1 A 56-year-old female patient with a brain metastasis originating from a papillary thyroid carcinoma treated with radiosurgery. Follow-up MR imaging 15 months later (top row, left) is consistent with stable disease according to RANO criteria for brain metastases. Most probably due to the lesion size, the corresponding FDG PET (top row, right) shows no increased metabolic activity. During the next 12 months, the size of contrast enhancement increased marginally (bottom row, left). Notwithstanding the small lesion size on anatomical MRI, the corresponding FDOPA PET (bottom row, right) shows clearly increased metabolic activity indicating brain metastasis relapse. ganglia and has also increasingly been used for imaging brain tumors. ${ }^{29}$ In the United States and western Europe FDOPA is approved for characterization of presynaptic dopaminergic activity in patients with Parkinsonian syndromes.

In both gliomas and BM, increased uptake of MET, FET, and FDOPA is due to large neutral LATs, which are overexpressed in neoplastic tissue (subtypes LAT1 and LAT2). ${ }^{17,30-32}$ Overexpression of LAT1, and therefore robustness of amino acid tracer uptake, closely correlates with malignant phenotype and proliferation of gliomas. ${ }^{33}$ Compared with MET and FDOPA, FET has high metabolic stability. After transport by LAT into neoplastic tissue, FET is not metabolized, ${ }^{25}$ whereas both MET and FDOPA show incorporation into protein, participation in other metabolic pathways, or metabolic degradation. ${ }^{34}$

Acquisition of dynamic FET PET data allows characterization of the temporal pattern of FET uptake by deriving a time-activity curve (TAC). It has been demonstrated that TAC parameters (eg, configuration, time-to-peak, slope) contain additional diagnostic information, which may be particularly valuable in the differentiation of $\mathrm{BM}$ recurrence from radiation-induced changes. ${ }^{35-37}$ Similarly, the ability of dynamic FET PET to distinguish recurrent glioma from radiation-induced treatment effect has also been described. ${ }^{38,39}$ Dynamic FET PET imaging is also helpful for glioma grading 40,41 and for determining the prognosis of untreated gliomas. ${ }^{42,43}$ Such utility has yet to be observed for dynamic MET or FDOPA PET.44,45

Lastly, the amino acid PET tracer $\alpha-{ }^{11} \mathrm{C}$-methyl-Ltryptophan (AMT) has recently been employed for brain tumor imaging in some centers. ${ }^{46}$ However, despite promising results in terms of differential diagnosis in patients with newly diagnosed brain tumors, including BM, the current literature is relatively small. ${ }^{47}$

\section{Other PET Tracers}

Only a few studies have used non-FDG and non-amino acid PET tracer imaging in patients with BM. Tracers such as ${ }^{18} \mathrm{~F}$-sodium fluoride $\left({ }^{18} \mathrm{~F}-\mathrm{NaF}\right), \quad 3^{\prime}$-deoxy- $3^{\prime}-{ }^{18} \mathrm{~F}-$ fluorothymidine ( $\left.{ }^{18} \mathrm{~F}-\mathrm{FLT}\right),{ }^{82}$ rubidium, as well as PET tracers targeting the endothelial prostate-specific membrane antigen (PSMA) have been used mostly for BM visualization and the assessment of treatment response. ${ }^{48-54}$ Choline derivatives (eg, ${ }^{18} \mathrm{~F}$-choline), which are in use for the diagnosis of recurrent prostate cancer, have also been reported to label BM. ${ }^{55,56}$ Animal studies have found that PET imaging using agents targeting the mitochondrial translocator protein (TSPO), which is upregulated on activated microglia, may be helpful for BM detection at an early stage of development. ${ }^{57}$ Despite promising results, experience with these tracers is based mainly on single cases in patients with BM, and their usefulness needs to be confirmed in larger studies.

\section{Clinical Applications for PET Imaging in Patients with Brain Metastasis}

\section{Identification of Newly Diagnosed and Untreated Brain Metastasis Using FDG and Amino Acid PET}

Although conventional MRI is the method of choice for the detection of $\mathrm{BM}$, some centers include the head for wholebody FDG PET/CT staging examinations of cancer patients. 
However, the value of this procedure is highly questionable based on the limited sensitivity of FDG PET for brain tumors related to the physiologically high levels of glucose metabolism in healthy brain parenchyma. ${ }^{58,59}$ Indeed, a prospective study has shown that, in comparison to contrastenhanced standard MRI for intracranial staging in newly diagnosed lung cancer, FDG PET has poor sensitivity $(27 \%)$ for BM detection. ${ }^{60}$ Similarly, a recent meta-analysis including more than 900 patients found that contrast-enhanced MRI has a higher cumulative sensitivity (77\%) than FDG PET $(21 \%)$ for the diagnosis of BM in lung cancer patients. ${ }^{61}$

The increased expression of amino acid transporters in BM compared with healthy brain tissue renders radiolabeled amino acids suitable for PET imaging based on high tumor-to-background contrast. ${ }^{17}$ Compared with FDG PET, the sensitivity of amino acid PET using FET to depict larger ( $>1 \mathrm{~cm}$ in diameter) BM seems to be clearly higher (approximately $90 \%$ of BM were FET positive based on a maximum tumor/brain ratio $\geq 1.6){ }^{62}$ However, detection of lesions with $<1 \mathrm{~cm}$ diameter may be considerably inferior to that of MRI. For example, in a pilot study of patients with newly diagnosed and untreated BM which correlated FET uptake characteristics with MRI parameters, the sensitivity of standard MRI for the detection of BM was $100 \%{ }^{62}$ Currently, the most sensitive and commonly used imaging modality for the detection of BM remains thin-slice contrast-enhanced MRI.

- Amino acid PET using the tracer FET has higher diagnostic accuracy for the detection of BM than FDG PET (evidence level 2).

- FDG or amino acid PET is limited in detecting smaller metastases, particularly those less than $1 \mathrm{~cm}$ in diameter.

- The imaging modality of choice for the detection of BM is contrast-enhanced MRI.

\section{Differential Diagnosis of Newly Diagnosed and Untreated Brain Metastasis Using FDG and Amino Acid PET}

FDG PET is limited in its ability to differentiate BM from mimics such as glioblastoma: it has been shown that there is no significant difference in FDG standardized uptake values (SUVs) between these entities. ${ }^{63,64}$ Differentiation between CNS lymphoma and BM based on FDG PET is more promising, as lymphoma may be substantially more FDG avid than BM. ${ }^{63,64}$ Initial data suggest that SUVs of the radiolabeled amino acid AMT are lower in BM than in glioblastomas. ${ }^{47}$ Further studies are required to firmly establish the added value of PET ligands to differentiate various lesions that have similar MRI characteristics.

High levels of LAT expression in cancer cells have been reported to correlate with aggressive tumor features and a worse prognosis. ${ }^{65,66}$ LAT expression also appears to be higher in recurrent compared with newly diagnosed BM. ${ }^{17}$ However, there are no studies yet investigating the prognostic value of amino acid PET in patients with BM. Possible limitations include the observation that uptake intensity as well as LAT expression levels are highly variable, even in metastases of the same primary tumor type. ${ }^{65,66}$ Thus, the site of origin of BM cannot be based on amino acid PET findings. ${ }^{62}$
In contrast to glioma, the size and volume of a BM are usually well delineated on contrast-enhanced MRI. Thus, amino acid PET does not add valuable information for biopsy or treatment planning as has been found for newly diagnosed gliomas. ${ }^{67,68}$

- There is limited evidence to support the use of PET to distinguish between BM and high-grade glioma (evidence level 3).

- Evidence is lacking for the use of amino acid PET to determine prognosis in patients with BM.

\section{Differentiation of Radiation-Induced Changes from Brain Metastasis Recurrence Using FDG and Amino Acid PET}

Oncologists of all subspecialties are often confronted with the clinical problem of differentiating tumor recurrence from treatment-related changes following radiation therapy, and in particular after high-dose focal radiation (ie, radiosurgery or fractionated stereotactic radiation therapy). Currently, conventional MRI does not reliably differentiate local brain tumor recurrence or progression from radiation-induced changes including radiation necrosis. In gliomas, radiation necrosis usually manifests within 6-12 months after standard fractionated radiotherapy and occurs in approximately $5-25 \%$ of all treated patients. ${ }^{69,70}$ For patients with BM treated by radiosurgery, a similar rate of radiation necrosis (approximately 25\%) has been reported, ${ }^{71}$ although the incidence depends on the radiation dose and irradiated brain volume. Indeed, in some cases the risk of radiation necrosis may be as high as $50 \% .{ }^{71}$ It should be noted that this wide variation in reported incidence is also likely a consequence of varying definitions of treatment-related changes in retrospective studies, including clinical data such as whether the patient is symptomatic or not. Treatment-related changes represent a spectrum of pathophysiology that may be purely radiographic and lack associated symptoms, but also may be symptomatic, refractory to corticosteroids, and require neurosurgical or other intervention.

\section{FDG PET}

In recent years, FDG PET has been studied as an additional neuroimaging tool to differentiate treatment-related effects from true BM progression (Table 1). Unfortunately, these investigations included few patients and were limited by variations in methodology such as thresholds used for the differentiation of radiation-induced changes from $\mathrm{BM}$ recurrence. Perhaps as a result, the diagnostic performance of FDG PET varied considerably (range of sensitivity, 40-95\%; range of specificity, 50-100\%) (Table 1). Dual phase FDG PET may be superior to standard (single phase) scans ${ }^{72}$ but limited by long time intervals between scans (median time between FDG PET scans, 3.8 hours; range, $2-5.7$ hours), ${ }^{72}$ hampering routine clinical use. The diagnostic performance of FDG PET also seems to be inferior to several other imaging methods, such as MET PET, ${ }^{73}$ MRI-based perfusion imaging with arterial spin labeling, ${ }^{74}$ MR spectroscopy, ${ }^{75}$ and diffusion-weighted imaging ${ }^{73,76}$ (Table 1). 
Table 1 Overview of studies regarding the differentiation of radiation-induced changes from brain metastasis recurrence using FDG PET

\begin{tabular}{|c|c|c|c|c|c|c|c|}
\hline & $\begin{array}{l}\text { Chao et al, } \\
2001^{77}\end{array}$ & $\begin{array}{l}\text { Belohlavek } \\
\text { et al, 2003 }\end{array}$ & $\begin{array}{l}\text { Chernov } \\
\text { et al, } \\
2005^{75}\end{array}$ & $\begin{array}{l}\text { Horky } \\
\text { et al, } 2011^{72}\end{array}$ & $\begin{array}{l}\text { Lai } \\
\text { et al, 2015 }\end{array}$ & $\begin{array}{l}\text { Hatzoglou } \\
\text { et al, } 2016^{76}\end{array}$ & $\begin{array}{l}\text { Tomura } \\
\text { et al, } 2017^{73}\end{array}$ \\
\hline Patients, $n$ & 32 & 25 & 9 & 25 & 14 & 24 & 15 \\
\hline Lesions, $n$ & 36 & 57 & 9 & 27 & 14 & 26 & 18 \\
\hline Recurrent metastases, $n$ & 18 & 8 & 4 & 16 & 6 & 11 & 10 \\
\hline $\begin{array}{l}\text { Radiation-induced } \\
\text { changes, } n\end{array}$ & 18 & 49 & 5 & 11 & 8 & 15 & 8 \\
\hline $\begin{array}{l}\text { Histological confirmation } \\
\text { of diagnosis }\end{array}$ & $36 \%$ & $5 \%$ & $56 \%$ & na & $100 \%$ & $23 \%$ & $56 \%$ \\
\hline FDG PET method & $\begin{array}{l}20 \text { min } \\
\text { static scan, } \\
45-60 \text { min } \\
\text { p.i. }\end{array}$ & $\begin{array}{l}15 \text { min } \\
\text { static scan, } \\
35-40 \text { min p.i. }\end{array}$ & na & $\begin{array}{l}\text { dual phase PET; } \\
\text { median time be- } \\
\text { tween early and } \\
\text { late scan, } 3.8 \mathrm{~h}\end{array}$ & $\begin{array}{l}\text { static scan } \\
60 \text { min p.i., } \\
\text { scan duration } \\
\text { na }\end{array}$ & $\begin{array}{l}10 \text { min static } \\
\text { scan, } 60 \text { min } \\
\text { p.i. }\end{array}$ & $\begin{array}{l}\text { static scan } \\
60 \text { min p.i., } \\
\text { scan } \\
\text { duration na }\end{array}$ \\
\hline Additional imaging method & no & no & MRS & no & ASL & DCE PWI & $\begin{array}{l}\text { DWI MET } \\
\text { PET }\end{array}$ \\
\hline Sensitivity & $65 \%$ & $75 \%$ & $50 \%$ & $95 \%$ & $83 \%$ & $82 \%$ & $40 \%$ \\
\hline Specificity & $80 \%$ & $94 \%$ & $80 \%$ & $100 \%$ & $75 \%$ & $80 \%$ & $50 \%$ \\
\hline Accuracy & na & $91 \%$ & $67 \%$ & $96 \%$ & $79 \%$ & na & na \\
\hline Threshold $\left(\mathrm{TBR}_{\text {mean }}\right)$ & visually & visually & visually & $\begin{array}{l}\text { change of L/GM } \\
\text { ratios }>0.19 \text { over } \\
\text { time }\end{array}$ & $3.0\left(\mathrm{SUV}_{\max }\right)$ & $1.4\left(\mathrm{TBR}_{\max }\right)$ & $0.97\left(\mathrm{TBR}_{\max }\right)$ \\
\hline $\begin{array}{l}\text { Performance of FDG PET } \\
\text { compared with other } \\
\text { imaging method(s) }\end{array}$ & na & na & inferior & na & inferior & inferior & inferior \\
\hline
\end{tabular}

$\mathrm{ASL}=$ arterial spin labeling; $\mathrm{DCE} P \mathrm{PWI}=$ dynamic contrast-enhanced perfusion-weighted imaging; $\mathrm{DWI}=$ diffusion-weighted imaging; $\mathrm{FDG}={ }^{18} \mathrm{~F}-2-$ fluoro-2-deoxy-D-glucose; L/GM = lesion to gray matter ratio; MET = ${ }^{11} \mathrm{C}$-methyl-L-methionine; na = not available; MRS = single- and multi-voxel proton MR spectroscopy; p.i. = post-injection; $\mathrm{TBR}_{\text {mean } \max }=$ mean or maximum standardized uptake value of the lesion divided by the maximum standardized uptake value of the reference region; $\mathrm{SUV}_{\max }=$ maximum standardized uptake value

\section{Amino Acid PET}

Amino acid PET has also been investigated as an imaging modality to distinguish treatment effect from tumor in clinical practice (Table 2). It has been demonstrated that MET PET may differentiate recurrent BM from radiation-induced changes using an easily applicable semi-quantitative regions-of-interest analysis for the calculation of tumor/brain ratios. MET PET has demonstrated a sensitivity and specificity of $70-80 \%$ in differentiating treatment effect from recurrent tumor. ${ }^{79-81}$ It has also been shown that FDOPA PET is able to differentiate recurrent $B M$ from radiation-induced changes with high sensitivity and specificity $(81 \%$ and $84 \%$, respectively) 82 (Fig. 1). Another study has reported an accuracy of $91 \%$ for differentiating radiation-induced changes from BM progression after radiosurgery for FDOPA PET, outperforming perfusion MRI parameters $91 \%$ to $76 \%{ }^{83}$ A similar high diagnostic performance has also been demonstrated for FET PET; using tumor/ brain ratios and dynamic parameters, FET PET differentiated radiation-induced changes from locally recurrent BM with a sensitivity of $95 \%$ and specificity of $91 \% 35$ (Fig. 2). Dynamic FET PET studies in a larger number of patients demonstrated a sensitivity and specificity of 80-90\%. ${ }^{36,37}$ Furthermore, the diagnostic performance of amino acid PET seems to be superior to both FDG PET and MRI-based perfusion- and diffusion-weighted imaging $^{73,83}$ (Table 2). Across all available amino acid PET studies for this indication, histological confirmation of diagnosis (ie, BM recurrence or radiation injury) ranges $11-56 \%$ (Table 2). The cost-effectiveness of amino acid PET has been demonstrated in Europe for the differentiation of recurrent $\mathrm{BM}$ and radiation-induced changes ${ }^{84}$ and various other indications. ${ }^{85-87}$

Recent literature highlights the value of PET radiomics in assessing tumor phenotypes. ${ }^{89}$ Radiomics enables the high-throughput extraction of a large number of quantitative features from imaging of a standard modality (usually $\mathrm{MRI}$ and PET). ${ }^{90,91}$ One application of radiomics is the use of textural feature analysis which objectively and quantitatively describes intrinsic properties of tumors, particularly heterogeneity. Using FET PET, it has been demonstrated that radiomic textural feature analysis provides non-invasive quantitative information useful for distinguishing treatment-related changes from disease progression. ${ }^{92}$ Combined FET PET and MRI radiomics using textural features achieved a diagnostic specificity of more than $90 \% .^{93}$

- Amino acid PET is useful in distinguishing posttherapeutic reactive changes following radiotherapy from recurrent BM. Present studies consistently show high diagnostic accuracy (evidence level 2). 


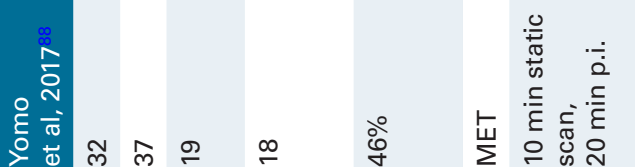 \\ 응 $\infty$ 审

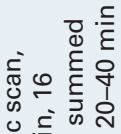 \\ รั

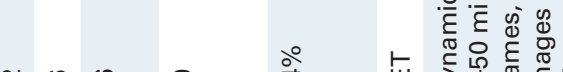

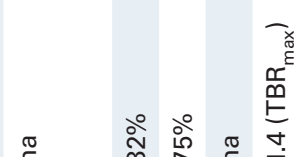

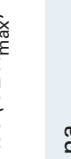

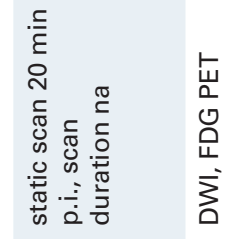
$0 \stackrel{\infty}{\circ}$ \&

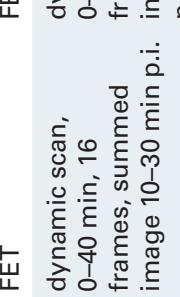

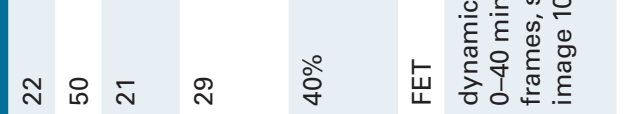

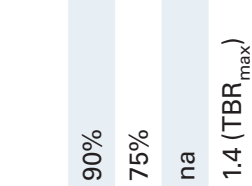

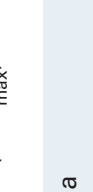

๘

@̊

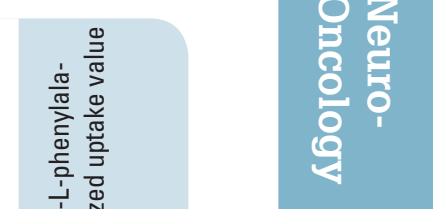

வ

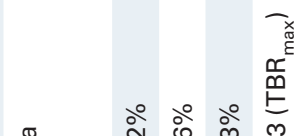

$\stackrel{\substack{x \\ m}}{\stackrel{\substack{x \\ m}}{m}}$

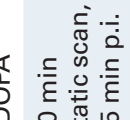

岳:

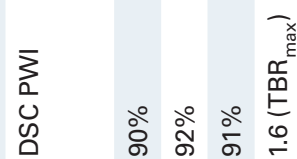

$\frac{1}{2}$

कृष

พ

$\stackrel{\circ}{\text { ¿ }}$

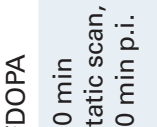

กల ๓

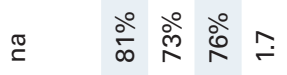

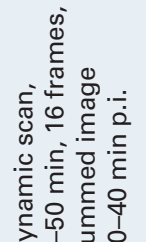

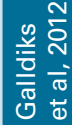

-

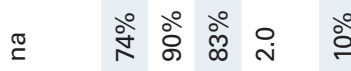

$\stackrel{0}{\check{2}}$

$\frac{\grave{\circ}}{\overline{\frac{0}{0}}}$

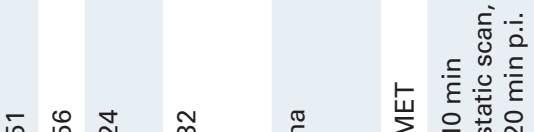

โ

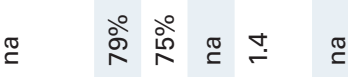

它

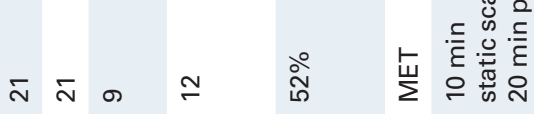

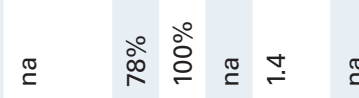



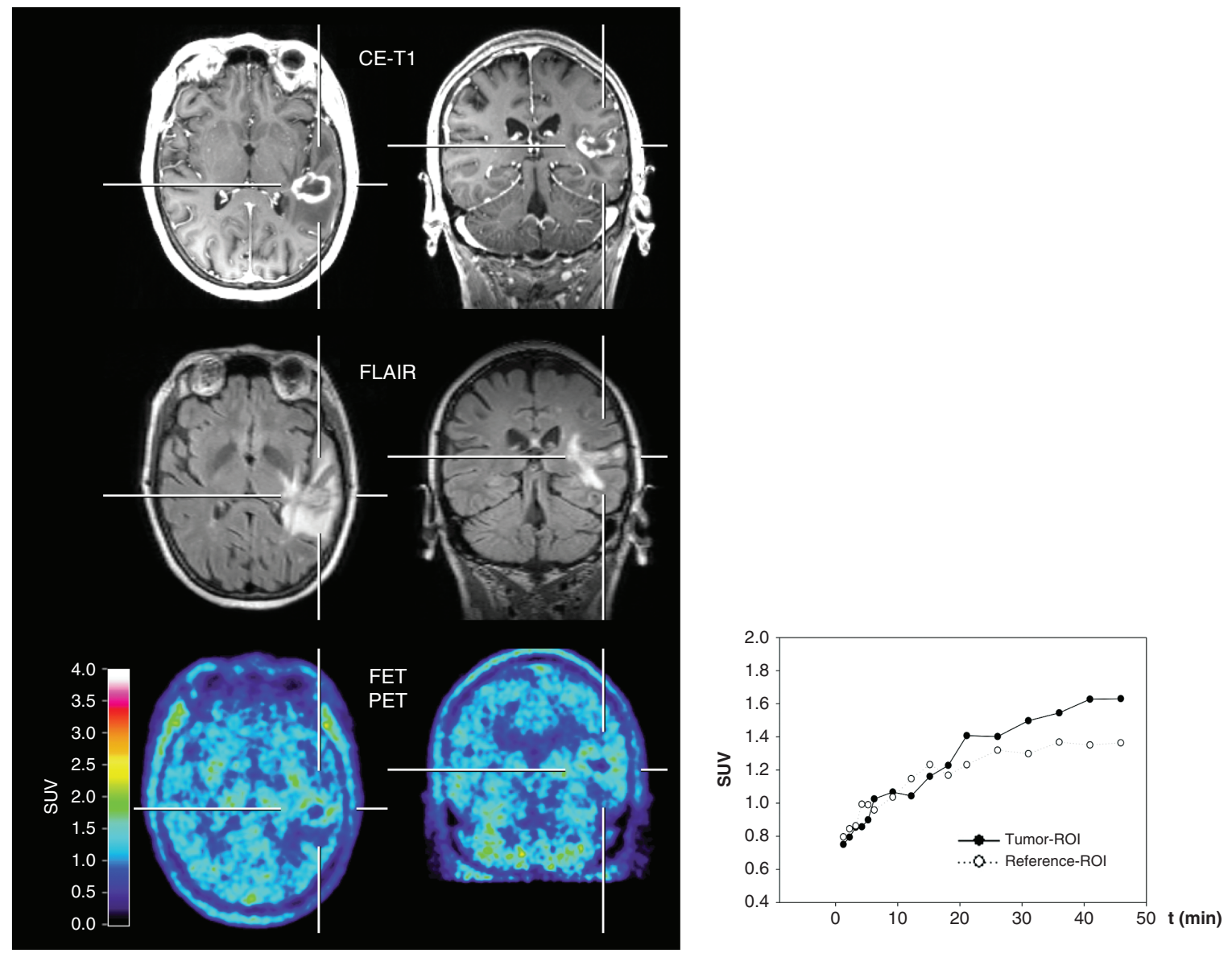

Fig. 2 A 50-year-old female patient with a brain metastasis secondary to non-small cell lung cancer underwent hybrid PET/MR imaging. Six months after stereotactic radiosurgery, MRI suggests tumor recurrence. In contrast, FET PET shows no increased metabolic activity $\left(T_{B R} \text { mean }_{2}=1.3\right)_{\text {, }}$ and the TAC shows a steadily increasing FET uptake, indicating radiation injury. The diagnosis was confirmed by subsequent hybrid PET/MR imaging 3 months later demonstrating improvement of imaging findings without a therapeutic intervention.

- FDG PET can also be used for this indication, but studies to date report highly variable diagnostic accuracy (evidence level 2).

- At present, direct comparisons of advanced MRI versus PET are limited. Amino acid PET may be more useful than advanced MRI, whereas FDG PET appears to be inferior (evidence level 3).

- When using PET for this indication, amino acid tracers should be preferred. Dynamic FET PET may further improve diagnostic accuracy.

\section{Differentiation of Treatment-Related Changes of Immunotherapy from Brain Metastasis Recurrence using FDG and Amino Acid PET}

Immuno-oncology is a rapidly developing therapeutic field with potential applications in CNS malignancies, particularly in patients with BM. ${ }^{94}$ Early phase studies have illustrated diagnostic challenges associated with the assessment of radiological changes in response to immunotherapy, wherein a subset of patients manifests delayed response to therapy or therapy-induced inflammation that mimics progressive disease. Following immunotherapy, long-term survival and tumor regression may occur after what was believed to represent initial disease progression or even after the appearance of new lesions. ${ }^{11}$ Pseudoprogression may occur in patients with BM treated with immunotherapy using checkpoint inhibitors such as cytotoxic $T$ lymphocyte-associated antigen 4 (eg, ipilimumab) and programmed cell death 1 receptor inhibitors (eg, pembrolizumab, nivolumab). ${ }^{11,12,95,96} \mathrm{~A}$ pilot study showed the potential of FET PET to identify pseudoprogression in patients with BM originating from melanoma treated with immune checkpoint inhibitors. ${ }^{97}$ Data on FDG PET for this indication are currently not available.

- At present, there is limited evidence of the potential benefit of amino acid PET for differentiating pseudoprogression from true disease progression following checkpoint inhibitor blockade (evidence level 3).

\section{Assessment of Treatment Response}

As stated above, standard MRI is limited in its ability to differentiate BM relapse from treatment-related effects such 

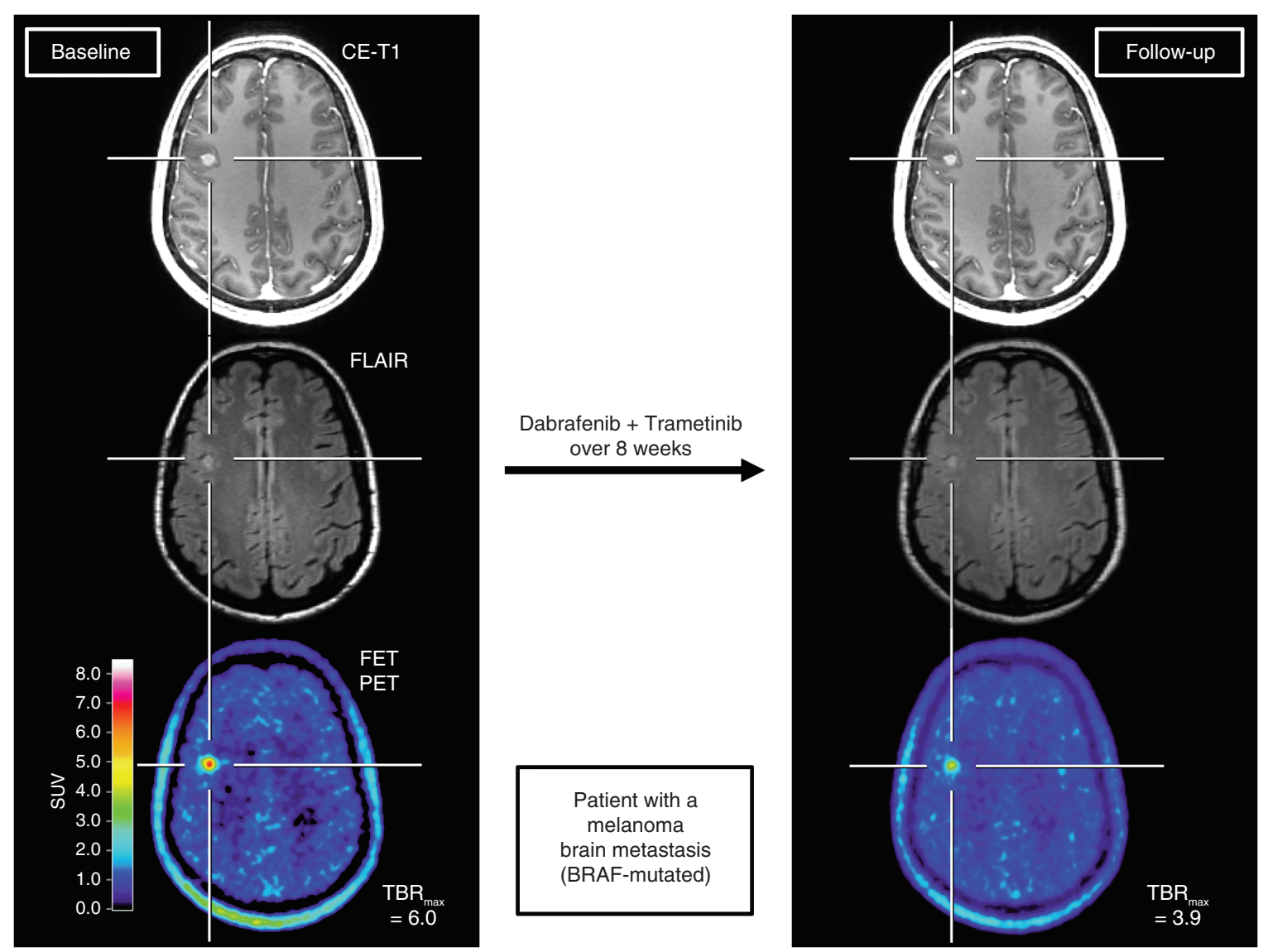

Fig. 3 A 45-year-old female patient with a brain metastasis secondary to a BRAF-mutated malignant melanoma treated with dabrafenib and trametinib. Comparison of contrast-enhanced MR and FET PET images at baseline (left column) and follow-up 8 weeks later (right column). At follow-up, a clear decrease of the tumor/brain ratios $(-35 \%)$ is observed, whereas the MRI shows no significant change of both the contrast enhancement and FLAIR signal defined as stable disease according to RANO criteria for brain metastases. The metabolic response was associated with an overall survival of 9 months after treatment initiation.

Table 3 Summary of recommendations

\begin{tabular}{|c|c|c|c|c|}
\hline & $\begin{array}{l}\text { Amino Acid PET } \\
\text { (MET, FET, FDOPA) }\end{array}$ & FDG PET & $\begin{array}{l}\text { Other } \\
\text { PET Tracers }\end{array}$ & $\begin{array}{l}\text { Oxford Level } \\
\text { of Evidence }\end{array}$ \\
\hline Identification of newly diagnosed BM & $(++)$ & - & na & 3 \\
\hline $\begin{array}{l}\text { Differential diagnosis of newly diagnosed } \\
\text { BM }\end{array}$ & $(++)$ & - & na & 3 \\
\hline $\begin{array}{l}\text { Differentiation of radiation-induced } \\
\text { changes from BM recurrence }\end{array}$ & $++^{*}$ & + & na & 2 \\
\hline $\begin{array}{l}\text { Differentiation of immunotherapy-related } \\
\text { changes from BM recurrence }\end{array}$ & $(++)$ & na & na & 3 \\
\hline Assessment of treatment response & $(++)$ & na & $(++)$ & 3 \\
\hline
\end{tabular}

as radionecrosis or pseudoprogression, all of which can induce contrast enhancement andT2/FLAIR hyperintensity. The use of FDG as a tracer for the assessment of treatment response in PET imaging is hampered by high physiologic brain uptake, limiting the discrimination between tumor and normal brain metabolic activity. ${ }^{14}$ Furthermore, in light of newer systemic treatment options such as targeted therapy and immunotherapy, tools which provide additional information on cellular physiology (eg, metabolism, proliferation) have become increasingly useful. 
The PET tracer FLT is an analog to the nucleoside thymidine and was developed as a PET agent to assess cellular proliferation by tracking the thymidine salvage pathway. ${ }^{98}$ Recently, FLT has been applied to assess response to the chemotherapeutic agent ANG1005 (a drug conjugate consisting of paclitaxel covalently linked to angiopep-2, designed to cross the blood-brain barrier) in patients with $\mathrm{BM}$ originating from breast cancer and was found to supplement the information derived from contrast-enhanced MRI by clarifying equivocal MRI findings. ${ }^{50}$

In BM from malignant melanoma being treated with targeted therapy and immunotherapy, a small study found in a subset of patients that metabolic responders may show a proliferative reduction on FLT PET despite apparent morphologic progression on standard MRI (ie, pseudoprogression). ${ }^{54}$

Studies evaluating amino acid PET for the assessment of treatment response are lacking. Theoretically, amino acid PET has the potential to add valuable information to standard MRI for the assessment of treatment response; validation in clinical studies is required. An illustrative example for this potential indication is presented in Fig. 3.

- Currently only preliminary evidence exists for a potential benefit of PET for the assessment of treatment response following systemic therapies (evidence level 3).

\section{Current Limitations and Future Perspective}

At present, the differentiation of radiation injury from BM recurrence using amino acid PET has been the most thoroughly investigated indication (Table 3), repeatedly demonstrating high diagnostic accuracy. However, it should be noted that these data were derived mainly from retrospective analyses performed in single centers, and diagnoses were not consistently confirmed histologically. Prospective multicenter studies are therefore needed to validate initial results of these proof-of-principle investigations. Challenges of prospective validation are several, including heterogeneity of patient population (ie, various originating cancers, number of BMs, and varying treatment regimens). Amino acid PET tracer availability and cost present additional obstacles.

Contrary to gliomas and transosseous meningiomas, the majority of BM can be easily delineated by conventional MRI.Thus, PET imaging does not add significant additional information. Detecting multiple, small BM remains a major clinical challenge, potentially impacting not only prognosis, but also treatment (ie, a shift from local treatment such as surgery or SRS to WBRT or systemic treatment options). Due to the limited spatial resolution of PET, miliary disseminated metastatic disease or leptomeningeal metastasis is challenging to assess and may be unapparent by current PET imaging. The still frequent use of FDG PET in the brain is of limited value due to poor lesion-to-background contrast, partially explaining disappointing results in screened cohorts. ${ }^{99}$ New PET tracers, such as TSPO ligands, might help to overcome this problem ${ }^{100}$ and may eventually assist in radiation treatment planning.
PET imaging in meningiomas using specific somatostatin receptor ligands such as ${ }^{68} \mathrm{Ga}$-DOTATATE PET has shown that tracer uptake may correlate with tumor grade as well as the likelihood of response to specific radionucleotide therapy. ${ }^{101,102}$ Intra-individual variation in patients with multiple lesions has been noted. ${ }^{103}$ By analogy, further investigations should aim to non-invasively image intra-individual heterogeneity in patients with multiple BM. Optimal patient management may benefit by improved and well-validated prognostic and predictive imaging markers derived from PET, as by the identification and quantification of target molecules for specific therapy (eg, epidermal growth factor receptor). ${ }^{104-106}$ Moreover, this could lead to early response markers of successful treatment that can be determined prior to changes in tumor size. Lastly, more specific PET tracers could potentially better identify BM primary cancer of origin.

By altering radioisotopes, PET ligands initially used for diagnostic imaging might also be instrumental for therapy. This concept of "theranostics" has already been introduced into the management of prostate cancer. ${ }^{107-109}$ Moreover, PET might help in the future to identify drug delivery into tumor tissue and provide imaging-based data on inter- and intra-individual variability of tumor drug concentration, thereby permitting more relevant information for patient selection and therapy tailoring. ${ }^{110,111}$

Another methodological innovation which could advance research in patients with BM is the increasing availability of hybrid PET/MR scanners, allowing the simultaneous acquisition of both imaging modalities. For example, the acquisition of dynamic FET PET, contrast-enhanced structural and perfusion-weighted MRI, and other advanced MRI sequences such as MR spectroscopy and functional MRI in a single session ("one-stop shop") can now be performed. Besides optimizing co-registration of various imaging modalities, this technology appears particularly attractive for BM patients with poor clinical condition by considerably reducing scanning time and avoiding exposure to additional radiation dose associated with a PET/CT scan. From a research perspective, hybrid PET/ MR technology provides a convenient platform for comparative imaging studies using amino acid PET and advanced MR imaging, ideally corroborated with neuropathology.

\section{Funding}

None.

Conflict of interest statement. All authors report no conflicts of interest related to the present work.

\section{References}

1. Ostrom OT, Wright CH, Barnholtz-Sloan JS. Brain metastases: epidemiology. Handb Clin Neurol. 2018;149:27-42. 
2. Soffietti R, Abacioglu U, Baumert B, et al. Diagnosis and treatment of brain metastases from solid tumors: guidelines from the European Association of Neuro-Oncology (EANO). Neuro Oncol. 2017;19(2):162-174.

3. Venur VA, Karivedu V, Ahluwalia MS. Systemic therapy for brain metastases. Handb Clin Neurol. 2018;149:137-153.

4. Wang TJC, Brown PD. Brain metastases: fractionated whole-brain radiotherapy. Handb Clin Neurol. 2018;149:123-127.

5. Thon N, Kreth FW, Tonn JC. The role of surgery for brain metastases from solid tumors. Handb Clin Neurol. 2018;149:113-121.

6. Langen KJ, Galldiks N, Hattingen E, Shah NJ. Advances in neuro-oncology imaging. Nat Rev Neurol. 2017;13(5):279-289.

7. Langen KJ, Galldiks N. Update on amino acid PET of brain tumours. Curr Opin Neurol. 2018;31(4):354-361.

8. Pope WB. Brain metastases: neuroimaging. Handb Clin Neurol. 2018;149:89-112.

9. Galldiks N, Law I, Pope WB, Arbizu J, Langen KJ. The use of amino acid PET and conventional MRI for monitoring of brain tumor therapy. Neuroimage Clin. 2017;13:386-394.

10. Camidge DR, Lee EQ, Lin NU, et al. Clinical trial design for systemic agents in patients with brain metastases from solid tumours: a guideline by the Response Assessment in Neuro-Oncology brain metastases working group. Lancet Oncol. 2018;19(1):e20-e32.

11. Okada $H$, Weller $M$, Huang $R$, et al. Immunotherapy response assessment in neuro-oncology: a report of the RANO working group. Lancet Oncol. 2015;16(15):e534-e542.

12. Wolchok JD, Hoos A, O'Day S, et al. Guidelines for the evaluation of immune therapy activity in solid tumors: immune-related response criteria. Clin Cancer Res. 2009;15(23):7412-7420.

13. Lin NU, Lee EQ, Aoyama $\mathrm{H}$, et al; Response Assessment in NeuroOncology (RANO) group. Response assessment criteria for brain metastases: proposal from the RANO group. Lancet Oncol. 2015;16(6):e270-e278.

14. Albert NL, Weller M, Suchorska B, et al. Response assessment in neurooncology working group and European Association for Neuro-Oncology recommendations for the clinical use of PET imaging in gliomas. Neuro Oncol. 2016;18(9):1199-1208.

15. Galldiks N, Albert NL, Sommerauer M, et al. PET imaging in patients with meningioma-report of the RANO/PET group. Neuro Oncol. 2017;19(12):1576-1587.

16. Langen KJ, Watts C. Neuro-oncology: amino acid PET for brain tumours-ready for the clinic? Nat Rev Neurol. 2016;12(7):375-376.

17. Papin-Michault C, Bonnetaud C, Dufour M, et al. Study of LAT1 expression in brain metastases: towards a better understanding of the results of positron emission tomography using amino acid tracers. PLoS One. 2016;11(6):e0157139.

18. Herholz K, Langen KJ, Schiepers C, Mountz JM. Brain tumors. Semin Nucl Med. 2012;42(6):356-370.

19. Bergström $M$, Collins VP, Ehrin $E$, et al. Discrepancies in brain tumor extent as shown by computed tomography and positron emission tomography using [68Ga]EDTA, [11C]glucose, and [11C]methionine. J Comput Assist Tomogr. 1983;7(6):1062-1066.

20. Galldiks N, Langen KJ, Pope WB. From the clinician's point of viewwhat is the status quo of positron emission tomography in patients with brain tumors? Neuro Oncol. 2015;17(11):1434-1444.

21. Huang $\mathrm{C}, \mathrm{McC}$ Conathy $\mathrm{J}$. Radiolabeled amino acids for oncologic imaging. J Nucl Med. 2013;54(7):1007-1010.

22. Langen KJ, Hamacher K, Weckesser M, et al. 0-(2-[18F]fluoroethyl)-Ltyrosine: uptake mechanisms and clinical applications. Nucl Med Biol. 2006;33(3):287-294.
23. Wester HJ, Herz M, Weber W, et al. Synthesis and radiopharmacology of 0-(2-[18F]fluoroethyl)-L-tyrosine for tumor imaging. J Nucl Med. 1999;40(1):205-212.

24. Langen KJ, Tonn JC, Weller M, Galldiks N. Letter to the Editor: "The role of imaging in the management of progressive glioblastoma. A systematic review and evidence-based clinical practice guideline" [J Neurooncol 2014; 118:435-460]. J Neurooncol. 2014;120:665-666.

25. Langen KJ, Jarosch M, Mühlensiepen $\mathrm{H}$, et al. Comparison of fluorotyrosines and methionine uptake in F98 rat gliomas. Nucl Med Biol. 2003;30(5):501-508.

26. Weber WA, Wester HJ, Grosu AL, et al. 0-(2-[18F]fluoroethyl)-L-tyrosine and L-[methyl-11C]methionine uptake in brain tumours: initial results of a comparative study. Eur J Nucl Med. 2000;27(5):542-549.

27. Grosu AL, Astner ST, Riedel E, et al. An interindividual comparison of 0-(2-[18F]fluoroethyl)-L-tyrosine (FET)- and L-[methyl-11C]methionine (MET)-PET in patients with brain gliomas and metastases. Int J Radiat Oncol Biol Phys. 2011;81(4):1049-1058.

28. Swissmedic. Swiss agency for therapeutic products. J Swissmedic. 2014;13:651.

29. Becherer A, Karanikas G, Szabó M, et al. Brain tumour imaging with PET: a comparison between [18F]fluorodopa and [11C]methionine. Eur J Nucl Med Mol Imaging. 2003;30(11):1561-1567.

30. Okubo S, Zhen HN, Kawai N, Nishiyama Y, Haba R, Tamiya T. Correlation of L-methyl-11C-methionine (MET) uptake with L-type amino acid transporter 1 in human gliomas. J Neurooncol. 2010;99(2):217-225.

31. Wiriyasermkul P, Nagamori S, Tominaga H, et al. Transport of 3-fluoro-L$\alpha$-methyl-tyrosine by tumor-upregulated L-type amino acid transporter 1 : a cause of the tumor uptake in PET. J Nucl Med. 2012;53(8):1253-1261.

32. Youland RS, Kitange GJ, Peterson TE, et al. The role of LAT1 in (18) F-DOPA uptake in malignant gliomas. J Neurooncol. 2013;111(1):11-18.

33. Haining Z, Kawai N, Miyake K, et al. Relation of LAT1/4F2hc expression with pathological grade, proliferation and angiogenesis in human gliomas. BMC Clin Pathol. 2012;12:4.

34. Singhal T, Narayanan TK, Jain V, Mukherjee J, Mantil J. 11C-Lmethionine positron emission tomography in the clinical management of cerebral gliomas. Mol Imaging Biol. 2008;10(1):1-18.

35. Galldiks N, Stoffels G, Filss CP, et al. Role of O-(2-(18)F-fluoroethyl)-Ltyrosine PET for differentiation of local recurrent brain metastasis from radiation necrosis. J Nucl Med. 2012;53(9):1367-1374.

36. Ceccon G, Lohmann P, Stoffels G, et al. Dynamic 0-(2-18F-fluoroethyl)L-tyrosine positron emission tomography differentiates brain metastasis recurrence from radiation injury after radiotherapy. Neuro Oncol. 2017;19(2):281-288.

37. Romagna A, Unterrainer M, Schmid-Tannwald C, et al. Suspected recurrence of brain metastases after focused high dose radiotherapy: can [18F] FET- PET overcome diagnostic uncertainties? Radiat Oncol. 2016;11(1):139.

38. Galldiks N, Dunkl V, Stoffels G, et al. Diagnosis of pseudoprogression in patients with glioblastoma using 0-(2-[18F]fluoroethyl)-L-tyrosine PET. Eur J Nucl Med Mol Imaging. 2015;42(5):685-695.

39. Galldiks N, Stoffels G, Filss C, et al. The use of dynamic 0-(2-18Ffluoroethyl)-I-tyrosine PET in the diagnosis of patients with progressive and recurrent glioma. Neuro Oncol. 2015;17(9):1293-1300.

40. Calcagni ML, Galli G, Giordano A, et al. Dynamic 0-(2-[18F]fluoroethyl)L-tyrosine (F-18 FET) PET for glioma grading: assessment of individual probability of malignancy. Clin Nuc/ Med. 2011;36(10):841-847.

41. Albert NL, Winkelmann I, Suchorska B, et al. Early static (18)F-FET-PET scans have a higher accuracy for glioma grading than the standard 20-40 min scans. Eur J Nucl Med Mol Imaging. 2016;43(6):1105-1114.

42. Jansen NL, Suchorska B, Wenter V, et al. Prognostic significance of dynamic 18F-FET PET in newly diagnosed astrocytic high-grade glioma. J Nucl Med. 2015;56(1):9-15. 
43. Jansen NL, Suchorska B, Wenter V, et al. Dynamic 18F-FET PET in newly diagnosed astrocytic low-grade glioma identifies high-risk patients. $J$ Nucl Med. 2014;55(2):198-203.

44. Kratochwil C, Combs SE, Leotta K, et al. Intra-individual comparison of ${ }^{18} \mathrm{~F}-\mathrm{FET}$ and ${ }^{18} \mathrm{~F}-\mathrm{DOPA}$ in PET imaging of recurrent brain tumors. Neuro Oncol. 2014;16(3):434-440.

45. Moulin-Romsée G, D'Hondt E, de Groot T, et al. Non-invasive grading of brain tumours using dynamic amino acid PET imaging: does it work for 11C-methionine? Eur J Nucl Med Mol Imaging. 2007;34(12):2082-2087.

46. Juhasz C, Dwivedi S, Kamson DO, Michelhaugh SK, Mittal S. Comparison of amino acid positron emission tomographic radiotracers for molecular imaging of primary and metastatic brain tumors. Mol Imaging. 2014;13(6):1-16.

47. Kamson D0, Mittal S, Buth A, et al. Differentiation of glioblastomas from metastatic brain tumors by tryptophan uptake and kinetic analysis: a positron emission tomographic study with magnetic resonance imaging comparison. Mol Imaging. 2013;12(5):327-337.

48. Salgarello $M$, Lunardi $G$, Inno $A$, et al. 18F-NaF PET/CT imaging of brain metastases. Clin Nucl Med. 2016;41(7):564-565.

49. Gori S, Inno A, Lunardi G, et al. 18F-sodium fluoride PET-CT for the assessment of brain metastasis from lung adenocarcinoma. $J$ Thorac Oncol. 2015;10(8):e67-e68.

50. O'Sullivan CC, Lindenberg M, Bryla C, et al. ANG1005 for breast cancer brain metastases: correlation between 18F-FLT-PET after first cycle and MRI in response assessment. Breast Cancer Res Treat. 2016;160(1):51-59.

51. Lu Y. FDG and (82)Rb PET/MRI features of brain metastasis of breast cancer. Clin Nucl Med. 2015;40(6):494-495.

52. Chakraborty PS, Kumar R, Tripathi M, Das CJ, Bal C. Detection of brain metastasis with 68Ga-labeled PSMA ligand PET/CT: a novel radiotracer for imaging of prostate carcinoma. Clin Nucl Med. 2015;40(4):328-329.

53. Chan M, Hsiao E, Turner J. Cerebellar metastases from prostate cancer on 68Ga-PSMA PET/CT. Clin Nucl Med. 2017:42(3):193-194.

54. Nguyen NC, Yee MK, Tuchayi AM, Kirkwood JM, Tawbi H, Mountz JM. Targeted therapy and immunotherapy response assessment with F-18 fluorothymidine positron-emission tomography/magnetic resonance imaging in melanoma brain metastasis: a pilot study. Front Oncol. 2018:8:18

55. Imperiale A, Bergerat JP, Saussine C, Abu Eid M, Kehrli P, Namer IJ. Isolated cerebellar metastasis from prostate adenocarcinoma diagnosed by $18 \mathrm{~F}-$ fluorocholine PET/CT: a rare but not impossible complication. Eur J Nucl Med Mol Imaging. 2014;41(2):397-398.

56. Gizewska A, Witkowska-Patena E, Stembrowicz-Nowakowska Z, Buraczewska A, Dziuk M. Brain metastases in patient with prostate cancer found in 18F-choline PET/CT. Nucl Med Rev Cent East Eur. 2015;18(1):39-41.

57. O'Brien ER, Kersemans V, Tredwell M, et al. Glial activation in the early stages of brain metastasis: TSPO as a diagnostic biomarker. J Nuc/ Med. 2014:55(2):275-280

58. Manohar K, Bhattacharya A, Mittal BR. Low positive yield from routine inclusion of the brain in whole-body 18F-FDG PET/CT imaging for noncerebral malignancies: results from a large population study. Nucl Med Commun. 2013:34(6):540-543.

59. Nia ES, Garland LL, Eshghi N, Nia BB, Avery RJ, Kuo PH. Incidence of brain metastases on follow-up 18F-FDG PET/CT scans of non-small cell lung cancer patients: should we include the brain? J Nucl Med Technol. 2017:45(3):193-197.

60. Krüger $S$, Mottaghy FM, Buck AK, et al. Brain metastasis in lung cancer. Comparison of cerebral MRI and 18F-FDG-PET/CT for diagnosis in the initial staging. Nuklearmedizin. 2011;50(3):101-106.

61. Li $Y$, Jin G, Su D. Comparison of gadolinium-enhanced MRI and 18FDG PET/PET-CT for the diagnosis of brain metastases in lung cancer patients: a meta-analysis of 5 prospective studies. Oncotarget. 2017;8(22):35743-35749.

62. Unterrainer M, Galldiks N, Suchorska B, et al. 18F-FET PET uptake characteristics in patients with newly diagnosed and untreated brain metastasis. J Nucl Med. 2017;58(4):584-589.

63. Purandare NC, Puranik A, Shah $S$, et al. Common malignant brain tumors: can 18F-FDG PET/CT aid in differentiation? Nucl Med Commun. 2017;38(12):1109-1116.

64. Meric K, Killeen RP, Abi-Ghanem AS, et al. The use of 18F-FDG PET ratios in the differential diagnosis of common malignant brain tumors. Clin Imaging. 2015;39(6):970-974.

65. Kaira K, Oriuchi N, Imai H, et al. Prognostic significance of L-type amino acid transporter 1 expression in resectable stage I-III nonsmall cell lung cancer. Br J Cancer. 2008;98(4):742-748.

66. Yanagisawa $\mathrm{N}$, Ichinoe M, Mikami T, et al. High expression of L-type amino acid transporter 1 (LAT1) predicts poor prognosis in pancreatic ductal adenocarcinomas. J Clin Pathol. 2012;65(11):1019-1023.

67. Pafundi DH, Laack NN, Youland RS, et al. Biopsy validation of 18F-DOPA PET and biodistribution in gliomas for neurosurgical planning and radiotherapy target delineation: results of a prospective pilot study. Neuro Oncol. 2013;15(8):1058-1067.

68. Munck Af, Rosenschold P, Costa J, et al. Impact of [18F]-fluoro-ethyltyrosine PET imaging on target definition for radiation therapy of highgrade glioma. Neuro Oncol. 2015;17(5):757-763.

69. Shah AH, Snelling B, Bregy A, et al. Discriminating radiation necrosis from tumor progression in gliomas: a systematic review what is the best imaging modality? J Neurooncol. 2013;112(2):141-152.

70. Kumar AJ, Leeds NE, Fuller GN, et al. Malignant gliomas: MR imaging spectrum of radiation therapy- and chemotherapy-induced necrosis of the brain after treatment. Radiology. 2000;217(2):377-384.

71. Minniti G, Clarke E, Lanzetta G, et al. Stereotactic radiosurgery for brain metastases: analysis of outcome and risk of brain radionecrosis. Radiat Oncol. 2011:6:48

72. Horky LL, Hsiao EM, Weiss SE, Drappatz J, Gerbaudo VH. Dual phase FDG-PET imaging of brain metastases provides superior assessment of recurrence versus post-treatment necrosis. J Neurooncol. 2011;103(1):137-146.

73. Tomura N, Kokubun M, Saginoya T, Mizuno Y, Kikuchi Y. Differentiation between treatment-induced necrosis and recurrent tumors in patients with metastatic brain tumors: comparison among 11C-methionine-PET, FDG-PET, MR permeability imaging, and MRI-ADC-preliminary results. AJNR Am J Neuroradiol. 2017;38(8):1520-1527.

74. Lai G, Mahadevan A, Hackney D, et al. Diagnostic accuracy of PET, SPECT, and arterial spin-labeling in differentiating tumor recurrence from necrosis in cerebral metastasis after stereotactic radiosurgery. AJNR Am J Neuroradiol. 2015;36(12):2250-2255.

75. Chernov M, Hayashi M, Izawa M, et al. Differentiation of the radiationinduced necrosis and tumor recurrence after gamma knife radiosurgery for brain metastases: importance of multi-voxel proton MRS. Minim Invasive Neurosurg. 2005;48(4):228-234.

76. Hatzoglou V, Yang TJ, Omuro A, et al. A prospective trial of dynamic contrast-enhanced MRI perfusion and fluorine-18 FDG PET-CT in differentiating brain tumor progression from radiation injury after cranial irradiation. Neuro Oncol. 2016;18(6):873-880.

77. Chao ST, Suh JH, Raja S, Lee SY, Barnett G. The sensitivity and specificity of FDG PET in distinguishing recurrent brain tumor from radionecrosis in patients treated with stereotactic radiosurgery. Int J Cancer. 2001;96(3):191-197.

78. Belohlavek O, Simonova G, Kantorova I, Novotny J Jr, Liscak R. Brain metastases after stereotactic radiosurgery using the Leksell gamma knife: can FDG PET help to differentiate radionecrosis from tumour progression? Eur J Nucl Med Mol Imaging. 2003;30(1):96-100. 
79. Terakawa $Y$, Tsuyuguchi N, Iwai $Y$, et al. Diagnostic accuracy of 11C-methionine PET for differentiation of recurrent brain tumors from radiation necrosis after radiotherapy. J Nuc/ Med. 2008;49(5):694-699.

80. Tsuyuguchi N, Sunada I, Iwai $Y$, et al. Methionine positron emission tomography of recurrent metastatic brain tumor and radiation necrosis after stereotactic radiosurgery: is a differential diagnosis possible? $J$ Neurosurg. 2003;98(5):1056-1064.

81. Minamimoto $R$, Saginoya $T$, Kondo $C$, et al. Differentiation of brain tumor recurrence from post-radiotherapy necrosis with 11C-Methionine PET: visual assessment versus quantitative assessment. Plos One. 2015;10(7):e0132515.

82. Lizarraga KJ, Allen-Auerbach M, Czernin J, et al. (18)F-FDOPA PET for differentiating recurrent or progressive brain metastatic tumors from late or delayed radiation injury after radiation treatment. J Nucl Med. 2014;55(1):30-36.

83. Cicone F, Minniti G, Romano A, et al. Accuracy of F-DOPA PET and perfusion-MRI for differentiating radionecrotic from progressive brain metastases after radiosurgery. Eur J Nucl Med Mol Imaging. 2015;42(1):103-111.

84. Heinzel A, Müller D, Yekta-Michael SS, et al. 0-(2-18F-fluoroethyl)-L-tyrosine PET for evaluation of brain metastasis recurrence after radiotherapy: an effectiveness and cost-effectiveness analysis. Neuro Oncol. 2017;19(9):1271-1278.

85. Heinzel A, Müller D, Langen KJ, et al. The use of 0-(2-18F-fluoroethyl)-Ltyrosine PET for treatment management of bevacizumab and irinotecan in patients with recurrent high-grade glioma: a cost-effectiveness analysis. J Nuc/ Med. 2013;54(8):1217-1222.

86. Heinzel A, Stock S, Langen KJ, Müller D. Cost-effectiveness analysis of amino acid PET-guided surgery for supratentorial high-grade gliomas. $J$ Nucl Med. 2012;53(4):552-558.

87. Heinzel A, Stock S, Langen KJ, Müller D. Cost-effectiveness analysis of FET PET-guided target selection for the diagnosis of gliomas. Eur J Nucl Med Mol Imaging. 2012;39(7):1089-1096.

88. Yomo S, Oguchi K. Prospective study of ${ }^{11} \mathrm{C}$-methionine PET for distinguishing between recurrent brain metastases and radiation necrosis: limitations of diagnostic accuracy and long-term results of salvage treatment. BMC Cancer. 2017;17(1):713.

89. Kickingereder $\mathrm{P}$, Götz M, Muschelli J, et al. Large-scale radiomic profiling of recurrent glioblastoma identifies an imaging predictor for stratifying anti-angiogenic treatment response. Clin Cancer Res. 2016;22(23):5765-5771.

90. Peeken JC, Nüsslin F, Combs SE. "Radio-oncomics": the potential of radiomics in radiation oncology. Strahlenther Onkol. 2017;193(10):767-779.

91. Galldiks N, Langen KJ. Amino acid PET in neuro-oncology: applications in the clinic. Expert Rev Anticancer Ther. 2017;17(5):395-397.

92. Lohmann P, Stoffels G, Ceccon G, et al. Radiation injury vs. recurrent brain metastasis: combining textural feature radiomics analysis and standard parameters may increase 18F-FET PET accuracy without dynamic scans. Eur Radiol. 2017;27(7):2916-2927.

93. Lohmann P, Kocher M, Ceccon G, et al. Combined FET PET/MRI radiomics differentiates radiation injury from recurrent brain metastasis. Neuroimag Clin. 2018;20:537-542.

94. Parakh S, Park JJ, Mendis S, et al. Efficacy of anti-PD-1 therapy in patients with melanoma brain metastases. $\mathrm{Br} J$ Cancer. 2017;116(12):1558-1563.
95. Preusser M, Lim M, Hafler DA, Reardon DA, Sampson JH. Prospects of immune checkpoint modulators in the treatment of glioblastoma. Nat Rev Neurol. 2015;11(9):504-514.

96. Roth P, Valavanis A, Weller M. Long-term control and partial remission after initial pseudoprogression of glioblastoma by anti-PD-1 treatment with nivolumab. Neuro Oncol. 2017;19(3):454-456.

97. Kebir S, Rauschenbach L, Galldiks N, et al. Dynamic 0-(2-[18F] fluoroethyl)-L-tyrosine PET imaging for the detection of checkpoint inhibitor-related pseudoprogression in melanoma brain metastases. Neuro Oncol. 2016;18(10):1462-1464.

98. Shields AF, Grierson JR, Dohmen BM, et al. Imaging proliferation in vivo with [F-18]FLT and positron emission tomography. Nat Med. 1998;4(11):1334-1336.

99. Diaz ME, Debowski M, Hukins C, Fielding D, Fong KM, Bettington CS. Non-small cell lung cancer brain metastasis screening in the era of positron emission tomography-CT staging: current practice and outcomes. J Med Imaging Radiat Oncol. 2018;62(3):383-388.

100. Unterrainer M, Fleischmann DF, Lindner $S$, et al. Detection of cerebrospinal fluid dissemination of recurrent glioblastoma using TSPO-PET With 18F-GE-180. Clin Nucl Med. 2018;43(7):518-519.

101. Sommerauer M, Burkhardt JK, Frontzek K, et al. 68Gallium-DOTATATE PET in meningioma: a reliable predictor of tumor growth rate? Neuro Oncol. 2016;18(7):1021-1027.

102. Hänscheid $H$, Sweeney RA, Flentje M, et al. PET SUV correlates with radionuclide uptake in peptide receptor therapy in meningioma. Eur $\mathrm{J}$ Nucl Med Mol Imaging. 2012;39(8):1284-1288.

103. Seystahl K, Stoecklein V, Schüller U, et al. Somatostatin receptor-targeted radionuclide therapy for progressive meningioma: benefit linked to 68Ga-DOTATATE/-TOC uptake. Neuro Oncol. 2016;18(11):1538-1547

104. McKnight BN, Kuda-Wedagedara ANW, Sevak KK, et al. Imaging EGFR and HER3 through 89Zr-labeled MEHD7945A (duligotuzumab). Sci Rep. 2018;8(1):9043.

105. Wei W, Ni D, Ehlerding EB, Luo OY, Cai W. PET imaging of receptor tyrosine kinases in cancer. Mol Cancer Ther. 2018;17(8):1625-1636.

106. Cheng S, Jacobson O, Zhu G, et al. Correction to: PET imaging of EGFR expression using an 18F-labeled RNA aptamer. Eur J Nucl Med Mol Imaging. 2018;45(12):2245.

107. Kulkarni HR, Singh A, Langbein $T$, et al. Theranostics of prostate cancer: from molecular imaging to precision molecular radiotherapy targeting the prostate specific membrane antigen. $\mathrm{Br} \mathrm{J}$ Radiol. 2018;91(1091):20180308.

108. Kircher M, Herhaus P, Schottelius M, et al. CXCR4-directed theranostics in oncology and inflammation. Ann Nucl Med. 2018;32(8):503-511.

109. Werner RA, Bundschuh RA, Bundschuh $L$, et al. Molecular imaging reporting and data systems (MI-RADS): a generalizable framework for targeted radiotracers with theranostic implications. Ann Nucl Med. 2018;32(8):512-522.

110. Man F, Lammers T, T M de Rosales R. Imaging nanomedicinebased drug delivery: a review of clinical studies. Mol Imaging Biol. 2018;20(5):683-695

111. Albert $S$, Arndt C, Koristka $S$, et al. From mono- to bivalent: improving theranostic properties of target modules for redirection of UniCAR $\mathrm{T}$ cells against EGFR-expressing tumor cells in vitro and in vivo. Oncotarget. 2018;9(39):25597-25616. 